extremities, inappropriate laughter, and seizures. Seizures are generalized in $90 \%$ of patients. Deafness has been reported previously, but the abnormal auditory brain stem responses recorded in this study appear to be unique.

Eight sporadic cases of Angelman syndrome associated with chromosome 15q12 deletion are reported from the Central Hospital, and Kobato Gakuen, Aichi Prefectural Colony, Japan (Matsumoto A et al. Epilepsia 1992; 33: 1083). Angelman syndrome is included in the etiology of West,

Lennox-Gastaut and other infantile-onset epileptic syndromes.

\title{
KETOGENIC DIET FOR INTRACTABLE SEIZURES
}

The efficacy of the ketogenic diet in 58 children with multiple seizure types resistant to antiepileptic drugs has been evaluated at the Johns Hopkins University School of Medicine, Baltimore. All patients had severe neurologic handicaps: mental retardation (84\%), cerebral palsy (45\%), and microcephaly (15\%). Mean age at diet initiation was 60 months (range 12-235 months). EEGs showed Lennox-Gastaut pattern (33\%), hypsarrhythmia (19\%), and focal activity (14\%). Antiepileptic drugs, including valproate, showed no adverse interaction. Seizures were completely controlled in $29 \%$, and reduced by half in $38 \%$. Diet was continued for an average of 24 months in those controlled and for 4 months in the unimproved group. Dietary benefit was not determined by seizure type. Renal stones required withdrawal of the diet in 2 patients. (Kinsman SL et al. Efficacy of the ketogenic diet for intractable seizure disorders: Review of 58 cases. Epilepsia Nov/Dec 1992; $\underline{33}$ :1132-1136). (Reprints: Dr SL Kinsman, Kennedy Krieger Institute,707 N Broadway, Baltimore, MD 21205).

COMMENT. Despite further demonstration of its efficacy and relative safety, the ketogenic diet is not a popular method of therapy among neurologists generally. Apart from the Mayo Clinic and Johns Hopkins, the diet is not frequently promoted in the training of pediatric neurologists and dieticians. Furthermore, the bias against fat in the diet in today's society adds to the difficulty in convincing mothers to become enthusiastic partners in the treatment program. Millichap JG at the Mayo Clinic (Epilepsia 1964; $\underline{5}: 239$ ) and Schwartz RM at the John Radcliffe Hospital, Oxford (Dev Med Child Neurol 1989; 31: 145) studied the metabolic effects of the diet and were unable to document any significant changes in blood lipid profiles using the classical diet. For an account of the mechanism of action of the diet, see Millichap JG. Progress in Pediatric Neurology, Chicago, PNB Publ, 1991, p 87-88.

\section{FELBAMATE IN LENNOX-GASTAUT SYNDROME}

The Felbamate Study Group report results in 73 patients ages 4 to 36

years with the Lennox-Gastaut syndrome, using a double-blind, placebo- 
controlled, add-on trial design. A maximum dosage of $45 \mathrm{mg} / \mathrm{kg} /$ body wt or $3600 \mathrm{mg} /$ day was titrated within 14 days. Felbamate-treated patients had a $34 \%$ decrease in frequency of atonic seizures, as compared with a $9 \%$ decrease in those receiving placebo. The total frequency of seizures was decreased by $19 \%$ in felbamate-treated patients, as compared with a $4 \%$ increase in the placebo group. Side-effects were similar in the two groups. Anorexia, vomiting, and somnolence occurred more frequently in the felbamate group, whereas diarrhea occurred more frequently in the placebo group. (Ritter FJ et al. Efficacy of felbamate in childhood epileptic encephalopathy (Lennox-Gastaut syndrome). N Engl J Med Jan 7 1993; $\underline{328:}$ : 29-33). (Reprints: Dr Frank J Ritter, Minnesota Epilepsy Group, 310 N Smith Ave, Ste 300, St Paul, MN 55102).

COMMENT. Felbamate (2-phenyl-1,3-propanediol dicarbamate) appears to be effective in the treatment of various types of seizures associated with the Lennox-Gastaut syndrome. Side effects are typically mild or moderate in severity and require no change in dosage. According to parental observations, the overall quality of life is also improved by increased alertness and verbal responsiveness.

\section{NEONATAL ELECTROGRAPHIC SEIZURES}

Preterm and full-term neonates with electrographically confirmed seizures were compared at the University of Pittsburgh School of Medicine. The incidence of seizures for all neonates admitted to a neonatal ICU was $2.3 \%$. Of 92 neonates with EEG-confirmed seizures seen over a 4 year period, 62 were preterm and 30 were full-term. Seizure frequency of preterm 30 wk neonates was $3.9 \%$ and significantly higher than that of older neonates. Clinical seizures were noted in $45 \%$ of preterm and $53 \%$ of full-term neonates. Seizures were subtle in $71 \%$ and $68 \%$, respectively. Ischemic brain lesions were diagnosed in $39 \%$ of preterm compared to $77 \%$ of full-term neonates. Intraventricular hemorrhage occurred in $45 \%$ of preterm and only in $3 \%$ of full-term neonates. Mortality was higher in the preterm than in the term populations. (Scher MS et al. Electrographic seizures in preterm and full-term neonates: Clinical correlates, associated brain lesions, and risk for neurologic sequelae. Pediatrics Jan 1993; 91: 128-134). (Reprints: Dr Mark S Scher, MageeWomens Hospital, Developmental Neurophysiology Laboratory, 300 Halket St, Pittsburgh, PA 15213).

COMMENT. More than $50 \%$ of this cohort had only electrographic expression of seizures. A later onset of seizures in preterm neonates suggests adverse medical complications after birth. One cannot always rely on simultaneous electrographic confirmation of seizures since $16 \%$ of patients exhibit electroclinical dissociation. 